\title{
The predictive value of inflammatory markers for pathological response of ipsilateral supraclavicular lymph nodes and for prognosis in breast cancer after neoadjuvant chemotherapy
}

\author{
Shaoqing Liu, Jing Fang, Dechuang Jiao, Zhenzhen Liu \\ Department of Breast surgery, Breast Cancer Center, Affiliated Cancer Hospital of Zhengzhou University, Zhengzhou, China \\ Contributions: (I) Conception and design: Z Liu; (II) Administrative support: Affiliated Cancer Hospital of Zhengzhou University; (III) Provision of \\ study materials or patients: Z Liu; (IV) Collection and assembly of data: S Liu, J Fang, D Jiao; (V) Data analysis and interpretation: S Liu, Z Liu; (VI) \\ Manuscript writing: All authors; (VII) Final approval of manuscript: All authors. \\ Correspondence to: Zhenzhen Liu. Department of Breast surgery, Breast Cancer Center, Affiliated cancer Hospital of Zhengzhou University, \\ Zhengzhou 450008, China. Email: liuzhenzhen73@126.com.
}

Background: Inflammatory tumor microenvironment is closely related to cancer. In this study, we mainly
explore the predictive value of inflammatory markers for pathological response of ipsilateral supraclavicular
lymph nodes (ISLN) and for prognosis in breast cancer with ISLN metastasis after neoadjuvant
chemotherapy (NAC).

Methods: In this study, 117 breast cancer patients with ISLN metastasis were collected from the Affiliated Hospital of Zhengzhou University. The best cut-off value was determined by using the receiver operating characteristics (ROC) curve. Chi-square test and binary Logistic regression were used to analyze the correlation between clinical pathological data and pathological response of ISLN and to determine independent predictors. Correlation analysis between inflammatory markers and prognosis used timedependent COX regression.

Results: The pathological complete response (pCR) rate of ISLN after NAC was 64.4\%. Multivariate analysis showed that breast pCR (OR 9.67, 95\% CI: 2.64-35.31, P<0.01) was an independent predictor of ISLN pathological response after NAC. After a median follow-up of 25 months, multivariate time-dependent COX results showed that higher platelet levels were correlated with poor disease-free survival (DFS) (HR 1.008, 95\% CI: 1.001-1.015, P=0.028). Meanwhile, menopausal status (HR 0.35, 95\% CI: 0.15-0.79, P=0.01) and supraclavicular pCR (HR 0.33, 95\% CI: 0.15-0.77, P=0.01) were also independent predictors of DFS.

Conclusions: Peripheral blood inflammatory markers have limited predictive value for pathological response of ISLN after NAC for breast cancer. High platelet count is associated with poor prognosis of breast cancer patients with ISLN metastasis.

Keywords: Inflammatory markers; neoadjuvant chemotherapy (NAC); ipsilateral supraclavicular lymph nodes (ISLN); pathological complete response (pCR); prognosis

Submitted Mar 12, 2020. Accepted for publication Aug 28, 2020.

doi: $10.21037 /$ gs-20-341

View this article at: http://dx.doi.org/10.21037/gs-20-341

\section{Introduction}

Existing studies suggest that invasive breast cancer with ipsilateral supraclavicular lymph node (ISLN) metastasis without distant metastasis accounts for $1-4 \%$ of all breast cancers (1-3). In the case of suspected metastasis of ISLN, clinical diagnosis is usually made by core needle biopsy of ISLN. Breast cancer patients with ISLN metastasis have a higher risk of distant metastasis, with a 5-year DMFS of $14.5-22 \%(4,5)$. Brito et al. reported for the first time that the 5 -year overall survival rate of 
patients with breast cancer with ISLN metastasis after multimodal comprehensive treatment was $41.1 \%$, which was significantly better than that of patients with distant metastasis (6). This result makes breast cancer patients with ISLN metastasis classified as N3C stage in the sixth edition of AJCC staging system (7). At present, comprehensive therapy such as neoadjuvant chemotherapy (NAC), surgical resection, local radiotherapy, etc. are mostly used clinically for such patients (8-11). Inflammation plays an important role in the occurrence and development of cancer (12). Tumor microenvironment consists of cells of mesenchymal and hematopoietic origin and non-cell components (13). The cellular components include fibroblasts, mesenchymal stem cells, epithelial cells, lymphoid and myeloid cells, etc., while the noncellular components refer to extracellular matrix (13). Inflammatory tumor microenvironment can enhance the growth, invasiveness and stem cell characteristics of breast cancer tumor cells $(14,15)$. Inflammatory reaction can cause an increase in neutrophil count and a slight increase in platelet count (16). Previous studies have shown that peripheral blood inflammatory markers have important value in predicting breast pathological response and breast cancer prognosis after NAC for breast cancer (17-20). However, there are few reports on the predictive value of peripheral blood inflammatory markers for pathological response and prognosis of breast cancer patients with ISLN metastasis after NAC. In this study, we mainly explored the predictive value of peripheral blood inflammatory markers for pathological response and prognosis of ISLN after NAC for breast cancer. We present the following article in accordance with the REMARK reporting checklist (21) (available at http:// dx.doi.org/10.21037/gs-20-341).

\section{Methods}

\section{Enrolled patients}

This retrospective study included 117 breast cancer patients with simultaneous ISLN metastasis treated in the affiliated Cancer Hospital of Zhengzhou University from January 2012 to December 2019. At the same time, patients were divided into two cohorts, 85 cases and 90 cases respectively, according to the research purpose. Enroll criteria: (I) diagnosis of unilateral primary breast cancer at first visit; (II) biopsy confirmation of ISLN metastasis; (III) treatment measures at least include NAC, surgical resection, and radiotherapy.
Among them, in the cohort of studying supraclavicular pCR after NAC, patients should meet the standard neoadjuvant chemotherapy scheme (EC-T8, TEC6, TCbH6). In the cohort of studying prognosis of breast cancer with ISLN metastasis, patients should satisfy the whole chemotherapy process as the standard scheme (EC-T8, TEC6, TCbH6); (IV) Complete peripheral blood inflammatory marker data including platelets, neutrophils and lymphocytes were available at baseline and during treatment. NLR referred to peripheral blood neutrophil count divided by lymphocyte count, while PLR referred to peripheral blood platelet count divided by lymphocyte count. Exclusion criteria: (I) distant metastasis at first visit; (II) concomitant with other tumors or with a history of tumors.

\section{Clinicopathological data}

The clinical data of the patients were collected, including age at first diagnosis, menopausal status, clinical $\mathrm{T}$ stage, chemotherapy regimen and pathological response of mass, axillary lymph node and ISLN after NAC, and the expression of ER, PR and HER2 in biopsy specimens was detected by immunohistochemical method (Figure S1). Complete peripheral blood inflammatory marker data including platelets, neutrophils and lymphocytes at 5 time points including baseline, post neoadjuvant chemotherapy, pre-operation, pre-radiotherapy and post-radiotherapy. Finally, we used the outpatient registration system for follow-up and determined the disease-free survival (DFS) according to the criteria. pCR was defined as Miller-Payne grade 5 of tumor after NAC or only ductal carcinoma in situ (22). (Specimens of breast masses, axillary lymph nodes and supraclavicular lymph nodes were obtained by modified radical mastectomy and supraclavicular lymph node dissection, and their pathological response were determined according to this standard). DFS was defined as the interval between initial diagnosis and local recurrence, distant recurrence, or contralateral breast cancer. The study was conducted in accordance with the Declaration of Helsinki (as revised in 2013). This study was approved by the Ethics Committee board of the Affiliated Cancer Hospital of Zhengzhou University (No. 2019001). As this study was retrospectively designed, informed consent was waived by the Affiliated Cancer Hospital of Zhengzhou University.

\section{Statistical analysis}

SPSS 23.0 and R software were used for statistical 
analysis in this study. Since there was no effective cutoff value, the ROC curve was analyzed to determine the best cut-off value for diagnosing the pCR of the ISLN. The best cutoff value was the maximum value of the sum of sensitivity and specificity on the ROC curve. At the same time, the area under the curve was calculated. The correlation between clinical data and pathological response of ISLN was analyzed by bilateral chi-square test. Binary logistic regression was used to determine the independent predictors of pathological response of ISLN. Univariate and multivariate time-dependent COX models were used to determine the predictive value of peripheral blood inflammatory markers for prognosis. Among the univariate variables, the variables with $\mathrm{P}<0.1$ were selected into the multivariate analysis. There was statistical difference when the bilateral test $\mathrm{P}<0.05$.

\section{Result}

\section{Patient baseline characteristics}

According to the enroll criteria, 117 breast cancer patients with ISLN metastasis were identified and patients were divided into two cohorts, 85 cases and 90 cases respectively. In the cohort of studying supraclavicular pCR after NAC (Table 1), the age distribution at initial diagnosis ranged from 26 to 69 years (median 50). 44.4\% $(n=44)$ of the patients were premenopausal, and $77.8 \%(n=70)$ of the patients had cT1-2 tumor size at the time of diagnosis. After NAC, the pCR rate of ISLN was $64.4 \%(n=58)$, axillary lymph nodes was $47.8 \%(\mathrm{n}=43)$, and breast masses was $35.6 \%(n=32)$. The mean baseline platelet count of this cohort was $255.07 \pm 64.44\left(\times 10^{9} / \mathrm{L}\right)$, the mean neutrophil count was $3.77 \pm 1.83\left(\times 10^{9} / \mathrm{L}\right)$, the mean PLR count was $140.09 \pm 44.73$, and the mean NLR count was $2.01 \pm 0.93$. According to the analysis results of the ROC curve, the optimal cutoff values of platelets, neutrophils, PLR and NLR were 261.5, 3.36, 129.60 and 1.51 respectively (Table 2). According to the best cutoff value, we divided 90 patients into two groups (low value group vs. high value group). $42.2 \%(\mathrm{n}=38), 52.2 \%(\mathrm{n}=47), 53.3 \%(\mathrm{n}=48)$ and $68.9 \%(\mathrm{n}=62)$ patients had high platelet, neutrophil, PLR and NLR respectively. In the cohort of studying the correlation between peripheral blood inflammatory markers and prognosis (Table 3), the age distribution at initial diagnosis was from 19 to 64 years (median 48), 47.1\% ( $\mathrm{n}=40)$ of the patients were postmenopausal, and $72.9 \%(\mathrm{n}=62)$ of the patients had tumor size cT1-2 at initial diagnosis.

\section{Correlation between baseline characteristics and pathological response of ISLN}

Univariate analysis showed that pathological response of ISLN after NAC were correlated with axillary pCR $(\mathrm{P}<0.01)$, breast pCR $(\mathrm{P}<0.01)$, platelets $(\mathrm{P}=0.01)$, PLR $(\mathrm{P}=0.03)$. Multivariate analysis further confirmed that breast pCR (OR 9.67, 95\% CI: 2.64-35.31, $\mathrm{P}<0.01$ ) was an independent predictor of ISLN pathological response after NAC (Table 1).

\section{Correlation between peripheral blood inflammatory markers and prognosis}

After a median follow-up of 25 months (7-65 months), $29.4 \%(n=25)$ of the patients developed distant metastasis. In order to explore the relationship between peripheral blood inflammatory markers and prognosis, and to consider the effect of changes of peripheral blood inflammatory markers on prognosis during treatment, we adopted timedependent COX model, which included baseline, postneoadjuvant, pre-operation, pre-radiotherapy and postradiotherapy data of 5 clinically valuable time nodes, and corrected other confounding factors (Table 4). Univariate time-dependent COX regression analysis results confirmed that premenopausal status (HR 0.38, 95\% CI: 0.16-0.88, $\mathrm{P}=0.02$ ), non-sPCR group (HR 0.30, 95\% CI: 0.13-0.68, $\mathrm{P}<0.01$ ), high platelet (HR 1.01, 95\% CI: 1.00-1.02, $\mathrm{P}=0.03$ ), high PLR group (HR 1.002, 95\% CI: 1.000 $1.004, \mathrm{P}=0.03$ ) patients were associated with poor DFS. Multivariate time-dependent COX model showed that high platelets were associated with poor prognosis (HR 1.008, 95\% CI: 1.001-1.015, $\mathrm{P}=0.028$ ), menopausal status (HR 0.35, 95\% CI: 0.15-0.79, $\mathrm{P}=0.01$ ), supraclavicular pCR (HR 0.33, 95\% CI: $0.14-0.77, \mathrm{P}=0.01$ ) were independent predictors of DFS.

\section{Discussion}

Lymphatic drainage of breast was mainly axillary lymph node, supraclavicular lymph node was one of lymphatic drainage areas of breast, about $3.1 \%$ of which is directly injected into supraclavicular lymph node on the same side (23). Breast cancer patients with ISLN metastasis and no distant metastasis account for about $1-4.3 \%$ of all breast cancer patients, and often indicate poor prognosis (1-4). At present, NAC, surgical resection of the primary lesion, combined with local radiotherapy are mostly used for 
Table 1 Cohort baseline characteristics of studying supraclavicular pCR after NAC

\begin{tabular}{|c|c|c|c|c|c|c|c|}
\hline Variables & Total & Non-sPCR, n (\%) & sPCR, n (\%) & $\mathrm{P}$ & OR & $95 \% \mathrm{Cl}$ & $\mathrm{P}$ \\
\hline$<50$ & 44 & $17(38.6)$ & $27(61.4)$ & & & & \\
\hline$\geq 50$ & 46 & $15(32.6)$ & $31(67.4)$ & & & & \\
\hline Menopause & & & & 0.47 & - & - & - \\
\hline Yes & 46 & $18(39.1)$ & $28(60.9)$ & & & & \\
\hline Clinical T stage & & & & 0.32 & - & - & - \\
\hline T1-2 & 70 & $23(32.9)$ & $47(67.1)$ & & & & \\
\hline T3-4 & 20 & $9(45.0)$ & $11(55.0)$ & & & & \\
\hline Positive & 56 & $23(41.1)$ & 33 (58.9) & & & & \\
\hline PR & & & & 0.70 & - & - & - \\
\hline Negative & 39 & 13 (33.3) & $26(66.7)$ & & & & \\
\hline Positive & 51 & 19 (37.3) & $32(62.7)$ & & & & \\
\hline HER2 & & & & 0.27 & - & - & - \\
\hline Negative & 55 & $22(40.0)$ & $33(60.0)$ & & & & \\
\hline Positive & 35 & $10(28.6)$ & $25(71.4)$ & & & & \\
\hline bPCR & & & & $<0.01$ & 9.67 & $2.64-35.31$ & $<0.01$ \\
\hline Yes & 43 & 7 (16.3) & $36(83.7)$ & & & & \\
\hline Platelet & & & & 0.01 & - & - & - \\
\hline$\leq 261.5$ & 52 & $13(25.0)$ & $39(75.0)$ & & & & \\
\hline$>261.5$ & 38 & $19(50.0)$ & $19(50.0)$ & & & & \\
\hline Neutrophil & & & & 0.10 & - & - & - \\
\hline$\leq 3.36$ & 43 & $19(44.2)$ & $24(55.8)$ & & & & \\
\hline$>3.36$ & 47 & $13(27.7)$ & $34(72.3)$ & & & & \\
\hline PLR & & & & 0.03 & - & - & - \\
\hline$\leq 129.60$ & 42 & $10(23.8)$ & $32(76.2)$ & & & & \\
\hline$>129.60$ & 48 & $22(45.8)$ & $26(54.2)$ & & & & \\
\hline NLR & & & & 0.05 & - & - & - \\
\hline$\leq 1.51$ & 28 & $14(50.0)$ & $14(50.0)$ & & & & \\
\hline$>1.51$ & 62 & $18(29.0)$ & $44(71.0)$ & & & & \\
\hline
\end{tabular}


Table 2 Analysis of inflammation markers of breast cancer ROC curve

\begin{tabular}{lcccc}
\hline Variables & Cut-off value & AUC $(95 \%$ Cl) & Specificity & Sensitivity \\
\hline Platelet & 261.5 & $0.62(0.49-0.74)$ & 0.67 & 0.59 \\
Neutrophil & 3.36 & $0.54(0.41-0.67)$ & 0.59 & 0.59 \\
PLR & 129.60 & $0.54(0.42-0.66)$ & 0.53 & 0.72 \\
NLR & 1.51 & $0.53(0.40-0.67)$ & 0.44 & 0.76 \\
\hline
\end{tabular}

Table 3 Cohort baseline characteristics of studying prognosis of breast cancer with ISLN metastasis

\begin{tabular}{|c|c|}
\hline Variables & Total, n (\%) \\
\hline \multicolumn{2}{|l|}{ Age, years } \\
\hline$<50$ & 49 (57.6) \\
\hline$\geq 50$ & $36(42.4)$ \\
\hline \multicolumn{2}{|l|}{ Menopause } \\
\hline No & 45 (52.9) \\
\hline Yes & $40(47.1)$ \\
\hline \multicolumn{2}{|c|}{ Clinical T stage } \\
\hline T1-2 & $62(72.9)$ \\
\hline T3-4 & $23(27.1)$ \\
\hline \multicolumn{2}{|l|}{ ER } \\
\hline Negative & $30(35.3)$ \\
\hline Positive & $55(64.7)$ \\
\hline \multicolumn{2}{|l|}{ PR } \\
\hline Negative & $36(42.4)$ \\
\hline Positive & 49 (57.6) \\
\hline \multicolumn{2}{|l|}{ HER2 } \\
\hline Negative & $60(70.6)$ \\
\hline Positive & 25 (29.4) \\
\hline \multicolumn{2}{|l|}{ aPCR } \\
\hline No & $54(63.5)$ \\
\hline Yes & $31(36.5)$ \\
\hline \multicolumn{2}{|l|}{ bPCR } \\
\hline No & $66(77.6)$ \\
\hline Yes & $19(22.4)$ \\
\hline \multicolumn{2}{|l|}{ sPCR } \\
\hline No & $41(48.2)$ \\
\hline Yes & $44(51.8)$ \\
\hline
\end{tabular}

breast cancer patients with ISLN metastasis, but the clinical benefits of supraclavicular lymph node dissection are still controversial (8-11).

Neoadjuvant chemotherapy affects the treatment strategy of breast cancer by reducing the mass and reducing the tumor stage, and has become the first choice for the treatment of locally advanced breast cancer (24). Previous studies have shown that compared with the ISLN pCR group, the non-pCR group had a lower local control rate and a lower DFS rate (25). In this study, compared with the non-pCR group, the risk of recurrence in the ISLN pCR group was 0.67 times lower than that in the non-pCR group (HR 0.33, 95\% CI: 0.15-0.77, $\mathrm{P}=0.01$ ). Previous studies have shown that the pCR rate of axillary lymph nodes was higher than that of breast after NAC $(26,27)$. In this study, the pCR rate of axillary lymph node was $47.8 \%$, the pCR rate of breast was $35.6 \%$, and the pCR of ISLN was significantly increased to $64.4 \%$, which indicated that supraclavicular lymph nodes were more sensitive to NAC.

The interaction between tumor and inflammatory cytokine was involved in the occurrence and development of tumor. Compared with inflammatory cytokine, inflammatory markers (neutrophils, platelets, NLR, PLR) are routine examination items in clinical practice and have higher clinical practicability and economic benefits. Studies have found that host inflammatory response was related to the prognosis of various tumors $(12,28)$. Neutrophils had both tumor-promoting and anti-tumor activities, and their overall effect on the body depended on which activity was dominant (29). Neutrophils elaborated tumor-promoting activity mainly by inhibiting the activation of CD8+ T cells (30), inhibiting the function of natural killer cells (31), and participating in the formation of neutrophil extracellular trap (NET) (32). In addition, neutrophils can elaborate anti-tumor activity through direct tumor cytotoxicity and enhancement of anti-tumor immunity mediated by cytotoxic $\mathrm{T}$ lymphocytes $(33,34)$. 
Table 4 Time-dependent COX regression analysis of time-varying inflammatory markers

\begin{tabular}{|c|c|c|c|c|c|c|}
\hline Variable & $\mathrm{HR}$ & $95 \% \mathrm{Cl}$ & $\mathrm{P}$ & $\mathrm{HR}$ & $95 \% \mathrm{Cl}$ & $\mathrm{P}$ \\
\hline Menopausal status & 0.38 & $0.16-0.88$ & 0.02 & 0.35 & $0.15-0.79$ & 0.01 \\
\hline Clinical T stage & 1.54 & $0.68-3.48$ & 0.30 & & & \\
\hline ER & 0.84 & $0.38-1.86$ & 0.66 & & & \\
\hline HER2 & 0.76 & $0.29-2.01$ & 0.58 & & & \\
\hline aPCR & 0.80 & $0.34-1.93$ & 0.62 & & & \\
\hline bPCR & 0.56 & $0.16-1.95$ & 0.36 & & & \\
\hline sPCR & 0.30 & $0.13-0.68$ & $<0.01$ & 0.33 & $0.14-0.77$ & 0.01 \\
\hline PLR & 1.002 & $1.000-1.004$ & 0.03 & 1.001 & $0.999-1.003$ & 0.36 \\
\hline NLR & 0.99 & $0.92-1.07$ & 0.83 & & & \\
\hline
\end{tabular}

The results of this study showed that in breast cancer patients with ISLN metastasis, neutrophils showed no correlation with pathological response of ISLN after NAC $(\mathrm{P}=0.10)$, and the correlation with prognosis of such patients (HR 0.83, 95\% CI: 0.58-1.19, $\mathrm{P}=0.31$ ).

In the cohort of studying prognosis, platelet was the only inflammation marker related to prognosis. Platelet was an important component in tumor microenvironment and was related to the prognosis and metastasis of various cancers $(35,36)$. Platelets can promote breast cancer cell growth and angiogenesis through surface integrin $\alpha 2 \beta 1$ connection and Wnt- $\beta$-catenin activation (37). In addition, platelets can also promote epithelial to mesenchymal transformation of cancer cells and promote metastasis through direct signal transmission with cancer cells (38). Consistent with other cancer research results, the results of this study show that in breast cancer patients with ISLN metastasis, higher platelet levels were associated with poor DFS (HR 1.008, 95\% CI: $1.001-1.015, \mathrm{P}=0.028)$. Platelets did not show predictive value for supraclavicular pCR after NAC, but further subgroup analysis showed that supraclavicular pCR was more easily achieved in patients younger than 50 years old, premenopausal patients and patients with lower levels of platelets after NAC. This result was consistent with the results of the study prognosis cohort in this study (the results were not shown), but a larger sample size study was still needed to verify this result.
NLR and PLR were two indicators based on inflammatory response. Previous studies had shown that NLR and PLR were related to breast cancer prognosis and pathological response after NAC (17-20). However, no such correlation was found in this study. The analysis showed that the patients included in this study were locally advanced breast cancer with ISLN metastasis, which was later than the previous study. In addition, NLR and PLR were considered as time-dependent covariates in this study, and the previous study uses the time-dependent working curve to obtain the best cut-off value and divides them into two groups. These differences may result in different conclusion.

There were some limitations in this retrospective clinical study. Retrospective analysis of clinical data may lead to outcome bias. In addition, due to the strict restriction of chemotherapy regimens, the number of patients included in this study was relatively small, and the follow-up time was relatively short, so larger samples were still needed to explore this clinical problem.

\section{Conclusions}

In conclusion, this study explored the correlation between peripheral blood inflammatory markers and supraclavicular pCR after NAC for breast cancer with ISLN metastasis and the prognosis of such patients. Our study showed that breast 
pCR was an independent predictor of supraclavicular pCR (OR 9.67, 95\% CI: 2.64-35.31, $\mathrm{P}<0.01$ ), while peripheral blood inflammatory markers did not show predictive value for supraclavicular pCR. Higher platelet levels were associated with poor DFS (HR 1.008, 95\% CI: 1.001-1.015, $\mathrm{P}=0.028$ ), suggesting that inflammatory parameters may be important for prognosis of breast cancer patients with ISLN metastasis.

\section{Acknowledgments}

Funding: This work was supported by a grant from Henan province medical science and technology research project (SBGJ2018088).

\section{Footnote}

Reporting Checklist: The authors have completed the REMARK reporting checklist. Available at http://dx.doi. org/10.21037/gs-20-341

Data Sharing Statement: Available at http://dx.doi. org/10.21037/gs-20-341

Peer Review File: Available at http://dx.doi.org/10.21037/gs20-341

Conflicts of Interest: All authors have completed the ICMJE uniform disclosure form (available at http://dx.doi. org/10.21037/gs-20-341). The authors have no conflicts of interest to declare.

Ethical Statement: The authors are accountable for all aspects of the work in ensuring that questions related to the accuracy or integrity of any part of the work are appropriately investigated and resolved. The study was conducted in accordance with the Declaration of Helsinki (as revised in 2013). This study was approved by the Ethics Committee board of the Affiliated Cancer Hospital of Zhengzhou University (No. 2019001). As this study was retrospectively designed, informed consent was waived by the Affiliated Cancer Hospital of Zhengzhou University.

Open Access Statement: This is an Open Access article distributed in accordance with the Creative Commons Attribution-NonCommercial-NoDerivs 4.0 International License (CC BY-NC-ND 4.0), which permits the noncommercial replication and distribution of the article with the strict proviso that no changes or edits are made and the original work is properly cited (including links to both the formal publication through the relevant DOI and the license). See: https://creativecommons.org/licenses/by-nc-nd/4.0/.

\section{References}

1. Galper S, Recht A, Silver B, et al. Factors associated with regional nodal failure in patients with early stage breast cancer with 0-3 positive axillary nodes following tangential irradiation alone. Int J Radiat Oncol Biol Phys 1999;45:1157-66.

2. Recht A, Gray R, Davidson NE, et al. Locoregional failure 10 years after mastectomy and adjuvant chemotherapy with or without tamoxifen without irradiation: experience of the Eastern Cooperative Oncology Group. J Clin Oncol 1999;17:1689-700.

3. Chen SC, Chen MF, Hwang TL, et al. Prediction of supraclavicular lymph node metastasis in breast carcinoma. Int J Radiat Oncol Biol Phys 2002;52:614-9.

4. Bonotto M, Gerratana L, Poletto E, et al. Measures of outcome in metastatic breast cancer: insights from a realworld scenario. Oncologist 2014;19:608-15.

5. Dawood S, Broglio K, Buzdar AU, et al. Prognosis of women with metastatic breast cancer by HER2 status and trastuzumab treatment: an institutional-based review. J Clin Oncol 2010;28:92-8.

6. Brito RA, Valero V, Buzdar AU, et al. Long-term results of combined-modality therapy for locally advanced breast cancer with ipsilateral supraclavicular metastases: The University of Texas M.D. Anderson Cancer Center experience. J Clin Oncol 2001;19:628-33.

7. Singletary SE, Allred C, Ashley P, et al. Revision of the American Joint Committee on Cancer staging system for breast cancer. J Clin Oncol 2002;20:3628-36.

8. Grotenhuis BA, Klem TM, Vrijland WW. Treatment outcome in breast cancer patients with ipsilateral supraclavicular lymph node metastasis at time of diagnosis: a review of the literature. Eur J Surg Oncol 2013;39:207-12.

9. Liu XH, Zhang L, Chen B. A meta-analysis of the prognosis in patients with breast cancer with ipsilateral supraclavicular lymph node metastasis versus patients with stage IIIb/c or IV breast cancer. Chronic Dis Transl Med 2016;1:236-42.

10. Jung J, Kim SS, Ahn SD, et al. Treatment Outcome of Breast Cancer with Pathologically Proven Synchronous Ipsilateral Supraclavicular Lymph Node Metastases. J 
Breast Cancer 2015;18:167-72.

11. Nikpayam M, Uzan C, Rivera S, et al. Impact of radical surgery on outcome in locally advanced breast cancer patients without metastasis at the time of diagnosis. Anticancer Res 2015;35:1729-34.

12. Allen MD, Jones LJ. The role of inflammation in progression of breast cancer: Friend or foe? (Review). Int J Oncol 2015;47:797-805.

13. McCuaig R, Wu F, Dunn J, et al. The biological and clinical significance of stromal-epithelial interactions in breast cancer. Pathology 2017;49:133-40.

14. Deshmukh SK, Srivastava SK, Bhardwaj A, et al. Resistin and interleukin-6 exhibit racially-disparate expression in breast cancer patients, display molecular association and promote growth and aggressiveness of tumor cells through STAT3 activation. Oncotarget 2015;6:11231-41.

15. Deshmukh SK, Srivastava SK, Zubair H, et al. Resistin potentiates chemoresistance and stemness of breast cancer cells: Implications for racially disparate therapeutic outcomes. Cancer Lett 2017;396:21-9.

16. Balkwill F, Mantovani A. Inflammation and cancer: back to Virchow? Lancet 2001;357:539-45.

17. Cuello-López J, Fidalgo-Zapata A, LopezAgudelo L, et al. Platelet-to-lymphocyte ratio as a predictive factor of complete pathologic response to neoadjuvant chemotherapy in breast cancer. PLoS One 2018;13:e0207224.

18. Patel DA, Xi J, Luo J, et al. Neutrophil-to-lymphocyte ratio as a predictor of survival in patients with triplenegative breast cancer. Breast Cancer Res Treat 2019;174:443-52.

19. Takeuchi H, Kawanaka H, Fukuyama S, et al. Comparison of the prognostic values of preoperative inflammationbased parameters in patients with breast cancer. PLoS One 2017;12:e0177137.

20. Asano Y, Kashiwagi S, Onoda N, et al. Predictive Value of Neutrophil/Lymphocyte Ratio for Efficacy of Preoperative Chemotherapy in Triple-Negative Breast Cancer. Ann Surg Oncol 2016;23:1104-10.

21. McShane LM, Altman DG, Sauerbrei W, et al. Reporting recommendations for tumor marker prognostic studies (REMARK). J Natl Cancer Inst 2005;97:1180-4.

22. Ogston KN, Miller ID, Payne S, et al. A new histological grading system to assess response of breast cancers to primary chemotherapy: prognostic significance and survival. Breast 2003;12:320-7.

23. Blumgart EI, Uren RF, Nielsen PM, et al. Predicting lymphatic drainage patterns and primary tumour location in patients with breast cancer. Breast Cancer Res Treat 2011;130:699-705.

24. Berruti A, Amoroso V, Gallo F, et al. Pathologic complete response as a potential surrogate for the clinical outcome in patients with breast cancer after neoadjuvant therapy: a meta-regression of 29 randomized prospective studies. J Clin Oncol 2014;32:3883-91.

25. Huang EH, Strom EA, Valero V, et al. Locoregional treatment outcomes for breast cancer patients with ipsilateral supraclavicular metastases at diagnosis. Int J Radiat Oncol Biol Phys 2007;67:490-6.

26. Tadros AB, Yang WT, Krishnamurthy S, et al. Identification of Patients With Documented Pathologic Complete Response in the Breast After Neoadjuvant Chemotherapy for Omission of Axillary Surgery. JAMA Surg 2017;152:665-70.

27. Zhu J, Jiao D, Guo X, et al. Predictive factors and prognostic value of pathologic complete response of ipsilateral supraclavicular lymph nodes in breast cancer after neoadjuvant chemotherapy. Ann Transl Med 2019;7:666.

28. Templeton AJ, McNamara MG, Seruga B, et al. Prognostic role of neutrophil-to-lymphocyte ratio in solid tumors: a systematic review and meta-analysis. J Natl Cancer Inst 2014;106:dju124.

29. Sagiv JY, Michaeli J, Assi S, et al. Phenotypic diversity and plasticity in circulating neutrophil subpopulations in cancer. Cell Rep 2015;10:562-73.

30. Coffelt SB, Kersten K, Doornebal CW, et al. IL-17producing gammadelta $\mathrm{T}$ cells and neutrophils conspire to promote breast cancer metastasis. Nature 2015;522:345-8.

31. Spiegel A, Brooks MW, Houshyar S, et al. Neutrophils Suppress Intraluminal NK Cell-Mediated Tumor Cell Clearance and Enhance Extravasation of Disseminated Carcinoma Cells. Cancer Discov 2016;6:630-49.

32. Park J, Wysocki RW, Amoozgar Z, et al. Cancer cells induce metastasis-supporting neutrophil extracellular DNA traps. Sci Transl Med 2016;8:361ra138.

33. Finisguerra V, Di Conza G, Di Matteo M, et al. MET is required for the recruitment of anti-tumoural neutrophils. Nature 2015;522:349-53.

34. Singhal S, Bhojnagarwala PS, O'Brien S, et al. Origin and Role of a Subset of Tumor-Associated Neutrophils with Antigen-Presenting Cell Features in Early-Stage Human Lung Cancer. Cancer Cell 2016;30:120-35.

35. Suzuki K, Aiura K, Kitagou M, et al. Platelets counts closely correlate with the disease-free survival interval of pancreatic cancer patients. Hepatogastroenterology 
2004;51:847-53.

36. Long Y, Wang T, Gao Q, et al. Prognostic significance of pretreatment elevated platelet count in patients with colorectal cancer: a meta-analysis. Oncotarget 2016;7:81849-61.

37. Zuo XX, Yang Y, Zhang Y, et al. Platelets promote breast cancer cell MCF-7 metastasis by direct interaction: surface integrin alpha2beta1-contacting-mediated activation of Wnt-beta-catenin pathway. Cell Commun Signal 2019;17:142.

38. Labelle M, Begum S, Hynes RO. Direct signaling between platelets and cancer cells induces an epithelialmesenchymal-like transition and promotes metastasis. Cancer Cell 2011;20:576-90.
Cite this article as: Liu S, Fang J, Jiao D, Liu Z. The predictive value of inflammatory markers for pathological response of ipsilateral supraclavicular lymph nodes and for prognosis in breast cancer after neoadjuvant chemotherapy. Gland Surg 2020;9(5):1354-1362. doi: 10.21037/gs-20-341 


\section{Supplementary}

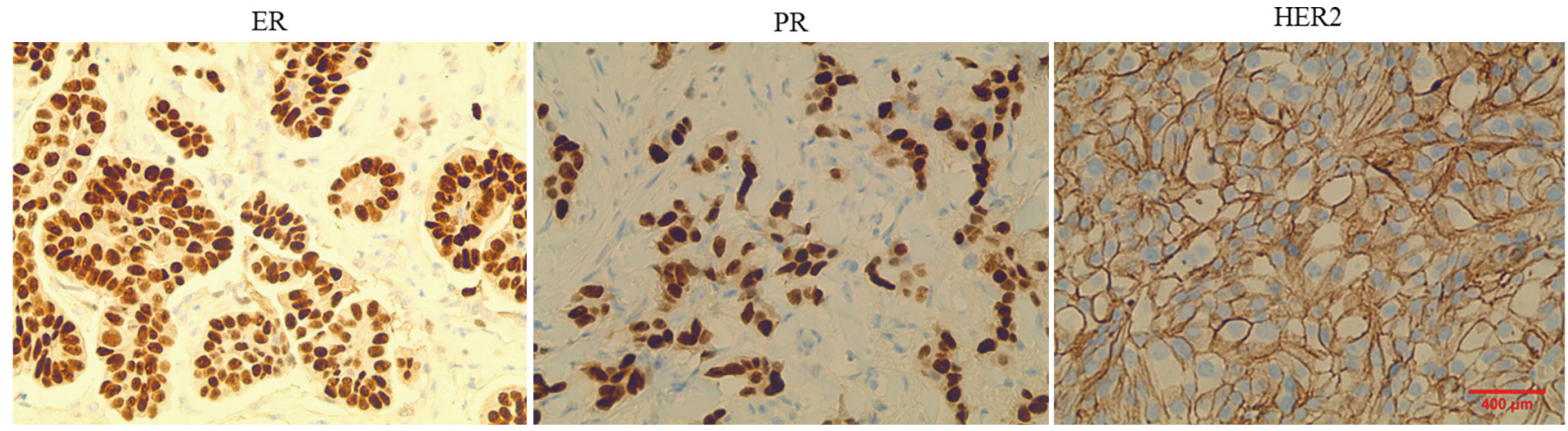

Figure S1 Immunohistochemical staining of ER, PR and HER 2 in breast cancer patients with ISLN metastasis. 